\title{
A Predictive Retransmission Scheme for Cooperative Routing in Intermittently Connected Sensor Networks
}

\author{
Md. Majharul Islam Rajib \\ Electrical and Computer Engineering \\ The University of North Carolina at Charlotte \\ 9201 University City Blvd. \\ Charlotte, NC 28223 \\ mrajib@uncc.edu
}

\author{
Asis Nasipuri \\ Electrical and Computer Engineering \\ The University of North Carolina at Charlotte \\ 9201 University City Blvd. \\ Charlotte, NC 28223 \\ anasipur@uncc.edu
}

\begin{abstract}
We consider energy harvesting sensor networks that are characterized by intermittent connectivity between sensor nodes due to random sleep and wake cycles caused by sporadic availability of energy sources. A retransmission strategy is developed that probabilistically predicts the best transmission interval between successive retransmissions in order to maximize the likelihood of successful packet delivery. We utilize this strategy for a cooperative routing approach to further improve the delay performance in such intermittently connected networks. An analytical model is formulated to study the delay characteristics of the proposed retransmission scheme. The performance of the proposed protocol in a multi-hop environment is evaluated through simulations. Results show that the retransmission scheme can provide significant performance improvements when compared to existing approaches.
\end{abstract}

\section{CCS Concepts}

-Networks $\rightarrow$ Link-layer protocols;

\section{Keywords}

Wireless sensor networks, energy harvesting, intermittently connected networks, cooperative relaying.

\section{INTRODUCTION}

Sensor networks powered by energy harnessed from the environment is a promising solution for long-term monitoring applications. However, many environmental energy sources such as mechanical vibrations, radio frequency, heat etc. are random and sporadic in nature $[13,15]$. Furthermore, they provide a small amount of energy at each harvesting event. The random availability of energy sources coupled with limitations of supply and storage make it difficult to operate the wireless sensor nodes continuously for prolonged periods. Consequently, sensor nodes may frequently have to

Permission to make digital or hard copies of all or part of this work for personal or classroom use is granted without fee provided that copies are not made or distributed for profit or commercial advantage and that copies bear this notice and the full citation on the first page. Copyrights for components of this work owned by others than ACM must be honored. Abstracting with credit is permitted. To copy otherwise, or republish, to post on servers or to redistribute to lists, requires prior specific permission and/or a fee. Request permissions from permissions@ acm.org.

ICDCN '16, January 04-07, 2016, Singapore, Singapore

(c) 2016 ACM. ISBN 978-1-4503-4032-8/16/01 . . $\$ 15.00$

DOI: http://dx.doi.org/10.1145/2833312.2833456 shut down all their power-hungry components such as the radio, and go into a deep-sleep mode until they recharge up to a certain level to become active again. This gives rise to an intermittently connected sensor network (ICSN), where nodes experience random and asynchronous outages [10].

Message delivery in such ICSNs poses several challenges. First, it is difficult to implement predetermined scheduling schemes due to the asynchronous nature of the sleep-awake periods of the nodes. Second, unlike non-rechargeable sensor networks, where an important objective is to optimize the usage of a finite amount of energy available in the batteries, the goal here is to wisely exploit the available energy resources. This is because the stored energy in such low capacity storage devices (e.g. super capacitors (SC)) may be lost due to leakage even without usage. Also, excess accumulation of harvested energy may be wasted due to the limited capacity of the energy storage device. Last but not least, multi-hop data delivery in ICSNs has to deal with the random delays or disruptions from hop to hop, which may not allow time-sensitive communications. To overcome these challenges in ICSNs, strategies such as cooperative routing can be deployed, which has been analyzed in greater detail in our earlier work [14].

In this paper, we focus on determining the optimal retransmission interval to further improve the transmission delay in ICSNs. We propose that, instead of performing backto-back retransmissions (as required by the X-MAC [4] or BOX-MAC [12]), it is more beneficial to use a retransmission interval for which the probability of the receiver being in active mode is maximum. The retransmission interval is a critical parameter in such low power energy harvesting nodes due to several reasons. First, transmission (as well as receptions) events are the biggest consumers of the energy in low power wireless sensor nodes. Hence, each energy harvesting event enables the source to attempt a limited number of transmissions to forward the packet to its parent node. Second, when energy arrival events are infrequent, the retransmission strategy must be based on the energy arrival rate to improve the probability of success. If the retransmission interval is too small, it will result in high energy wastage due to many unsuccessful retransmissions. On the other hand, an extremely high retransmission interval might miss the window of time when the receiver become active and result a longer average delay.

Leveraging these concepts, our objective in this paper is to enhance the delay performance of cooperative routing through a more judicious choice of packet retransmission 

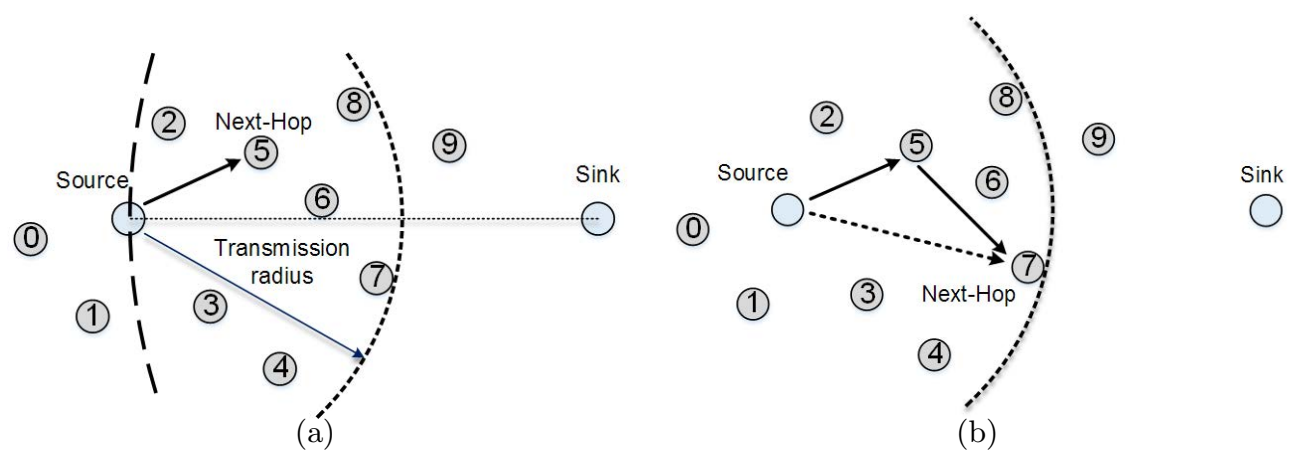

Figure 1: Operation of (a) opportunistic routing and (b) cooperative routing in an intermittently connected network.

times. We develop a probabilistic model to predict the optimal retransmission time for an intermittently connected wireless link, and then utilize the model to construct an optimum retransmission scheme using cooperative routing. We develop a mathematical formulation to estimate the expected delay, and use simulations to evaluate the delay characteristics of the proposed model.

The rest of the paper is organized as follows. Section 2 discusses related works. In section 3 , we present the formulation of the optimal retransmission interval and analysis of the delay over intermittently connected wireless links. Section 4 discusses the proposed scheme that utilizes optimal retransmission intervals in cooperative routing. In section 5 , we present performance evaluations from computer simulations, and in section 6 , we present the conclusions.

\section{RELATED WORK}

A commonly used approach for conserving energy in asynchronous wireless sensor networks is asynchronous duty-cycling, also know as low-power listening (LPL). However, effective LPL strategies typically require a series of back-to-back transmissions of short preambles (e.g. X-MAC) or copies of the data packets (e.g. BOX-MAC, ContikiMAC [6]). None of these approaches consider appropriate retransmission intervals between theses repeated transmissions. Authors in [16] proposed a predictive wake up mechanism using pseudo-random schedule generator, where a source can predict the receiver's wake up schedule by exchanging generator parameters. This is not applicable for ICSN, because any form of scheduling can not be guaranteed due to the randomness of energy availability.

Two broad classes of routing schemes have been explored for reducing the end-to-end transmission delay in such intermittently connected networks. The first is opportunistic routing $[7,9,18]$, where an active node anycasts its packet to a set of neighboring nodes who can provide routing progress. Nodes are assumed to be aware of their geographic locations. Any node within the set that receives the packet, forwards it to the next hop similarly. This type of routing does not require scheduling schemes, and it opportunistically utilizes available energy in the surroundings through anycasting. However, it has very minimal control over the routing path since there is no way to determine which node in the set is going to be the next-hop. Opportunistic rout- ing is illustrated in Fig. 1(a), where a packet transmitted by the source may be forwarded by one or more of nodes 2-8 that have the potential to provide routing progress towards the sink. These nodes are called the forwarder set of the source. Multiple methods have been proposed to choose this forwarder set. For instance, the authors of [5] considered the nodes within a $[-30,30]$ degree angle from the straight line connecting the source to the sink to be the forwarding set. The node within the forwarder set that first receives the packet acknowledges and forwards the packet to the next hop (node 5 in the example). A problem arises when more than one node in the forwarder set receives the packet, which would result in duplicate copies of the same packet to be forwarded to the sink. This might eventually lead to degraded performance due to energy wastage, especially in ICSNs that relies on ambient energy harvesting. Several approaches have been proposed to overcome this issue. Authors in $[7,11]$ split the forwarder set into a number of regions and assigned a priority to each region based on their potential routing progress. When multiple nodes in the forwarder set receive the packet, nodes from the highest priority regions declare themselves as next-hops first. Nodes from the lower priority zone discard the packet if they see that a node from a higher priority zone already declared itself as next-hop. However, even with this, the likelihood of forwarding multiple copies is not completely removed, since each priority region may consist of multiple nodes. Another approach presented in [9] requires immediate packet retransmission to inform the forwarding nodes that more than one node have received the packet.

The cooperative routing scheme is illustrated in Fig. 1(b). Here, a unicast routing approach is followed, where every node determines the next-hop through some least cost routing protocol. For example, in Fig. 1(b), source determines node 7 as the next-hop since that is the closest to sink. When source transmits a packet destined for node 7, common neighbors of source and 7 , such as node 5 , assists in reducing the delay in the process through cooperative relaying. In this scenario, if the source's transmission fails to reach 7 , but overheard by 5 (we call it the relay node), node 5 starts independently forwarding the packet on behalf of source. It may happen that node 5 might be active at the same time as 7, while the source is inactive. Since 5 is transmitting source's data packet, a faster packet delivery is possible in that case. 
Though cooperative routing by itself has a poorer delay performance in comparison to opportunistic routing, it provides several advantages that are beneficial in energy harvesting sensor networks. First, since cooperative routing leverages unicast routing, packet duplication is minimized. Therefore, it does not require any additional mechanisms to reduce packet duplications. Next, cooperative routing allows for more supervised and controlled routing. For example, in the illustration in Fig. 1(b), the source may choose node 1 as the next-hop to steer packet routing around node $2-8$ if it observes that nodes 2-8 are getting congested or constrained in energy resources. While the relative performance of cooperative and opportunistic schemes are subject to successful implementations of energy conserving strategies, here, our focus is on optimizing the retransmission interval for improving the delay performance in cooperative routing.

\section{PREDICTIVE RETRANSMISSION FOR INTERMITTENT WIRELESS LINKS}

In this section, we describe the system model in greater detail and develop the basis for our probabilistic prediction for retransmission period. We approach the problem by demonstrating that the optimum retransmission interval can be obtained from the available energy level at the node (battery life) and the energy harvesting rate. We then show that using the optimum retransmission interval for every retransmission attempt minimizes the average packet transmission delay over the intermittently connected wireless link. The idea is illustrated in Fig. 2. In Fig. 2 (a), the transmitter employs back-to-back retransmissions as required by popular schemes such as X-MAC, where the expectation is for the receiver to receive and acknowledge the packet whenever it wakes up. However, as illustrated in Fig. 2(a), this might lead to the transmitter to deplete its energy before it could reach the receiver and go to inactive (sleep) mode. We propose that instead of performing immediate retransmissions, it is more effective for the transmitter to retransmit at a time when the probability of the receiver waking up is predicted to be maximum (see Fig. 2(b)). This maximizes the probability of successful transmission in the transmitter's current active period and thereby reduces the risk of the transmitter going back to sleep prior to successfully transmitting the packet.

To verify this, a detailed delay analysis for both strategies is presented later. In the following, we develop a probabilistic model to predict the optimal waiting time between successive retransmissions based on current energy status of the transmitter node.

\subsection{Preliminaries}

We consider event monitoring sensor networks, where nodes periodically sample sensor data and report information to the sink through multi hop routing only when specific "interesting" events are detected. Some applications of these types of networks are volcanic activity monitoring [17], intrusion detection [2], and fire disaster management [3], to name a few. At the setup phase of the network, nodes gather route related data and slowly update the route information through 'hello' messages thereafter.

We consider that all nodes are static and they independently harvest energy from the environment. The harvested energy is stored in their respective SCs, and when the accu-
Table 1: Notations

\begin{tabular}{|c|l|}
\hline$E_{h}$ & Amount of energy in each harvest \\
\hline$E_{c}$ & Energy remaining in the storage \\
\hline$E_{t}$ & Required energy for a packet transmission \\
\hline$E_{r}$ & Required energy for a packet reception \\
\hline$E_{o}$ & Energy consumed in overhearing \\
\hline$E_{a}^{t}$ & Ambient consumption by transmitting node \\
\hline$E_{a}$ & Ambient consumption of a general node \\
\hline$P_{e}$ & Energy arrival probability \\
\hline$P_{o}$ & Probability of overhearing \\
\hline$P_{c}$ & Probability that the channel is error free \\
\hline$T_{w}$ & Waiting time before retransmission \\
\hline
\end{tabular}

mulated energy exceeds a certain threshold, they enter an active mode to perform regular activities such as periodic sampling, processing, and communication tasks. When the energy is depleted to such a level that nodes no longer can participate in transmissions or receptions, they go into a deep-sleep or inactive mode. Due to low energy availability, the stretch of the inactive period is assumed to be large compared to the active periods. Furthermore, both active and inactive states have random durations due to the randomness of the energy sources and energy consumption events such as reception and overhearing. Therefore, no node can schedule sleep wake cycles ahead of time. A random access MAC is assumed, where only one of several contending nodes gets to access the channel at any time, while others overhear/receive the packet.

\subsection{Energy Dynamics of a Sensor Node}

In order to predict the optimal retransmission time, it is essential to characterize the energy dynamics of a sensor node. Here we discuss the basic energy expenditure and intake behavior of a node. The energy stored in a sensor node's storage device changes due to primarily three causes. First, the energy level increases by $E_{h}$ for each "energy arrival" event, which happens when environmental energy is harvested. We assume that these are bursty events, typical of vibration energy harvesting from passing cars (e.g. on a bridge or roadside). We model the inter-arrival times of energy events to be exponentially distributed. Second, the energy level gets depleted due to radio events, which includes packet transmissions (consumes $E_{t}$ ), receptions (consumes $E_{r}$ ), or overhearing (consumes $E_{o}$ ). Finally, we consider that when a node is in active state, ambient events such as sensing, processing, and low power listening depletes its energy at a constant rate. To simplify, we term all these activities together as "ambient-activities", which requires an average "ambient consumption" of $E_{a}^{t}$ or $E_{a}$ energy for transmitter and receiver respectively. This spans throughout the active period. Fluctuations in node's energy level due to these activities are illustrated in the top of Fig. 3.

To make computations tractable, we consider a discrete time version of this continuous time model (bottom of Fig. $3)$. We assume that time is slotted and each slot is sufficiently small so that only one transmission can take place in a slot. The inter energy arrival time can then be represented by a Geometric distribution with a parameter $P_{e}$, where $P_{e}$ 


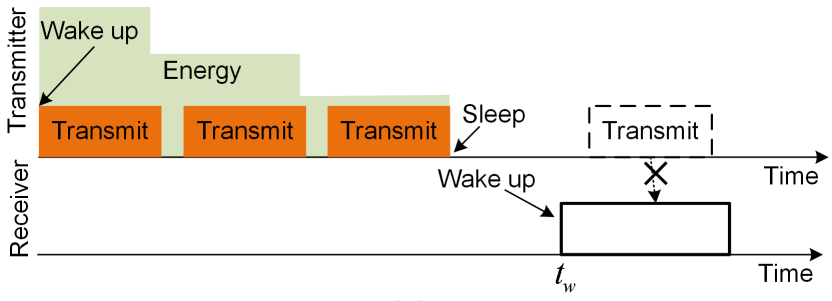

(a)

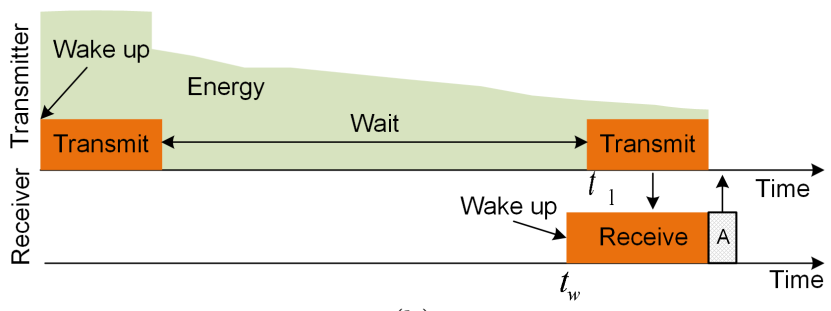

(b)

Figure 2: Illustration of (a) back-to-back and (b) predictive retransmissions.

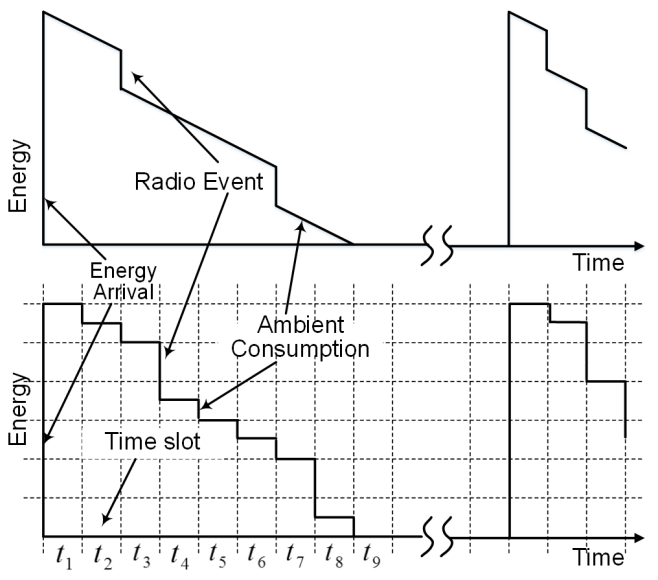

Figure 3: Continuous time representation (top) and its discrete time approximation (bottom) of the variation of energy level in a rechargeable sensor node.

represents the energy arrival probability at each slot. Considering that the energy arrival rate is small, i.e. the inter energy arrival times are long, the probability of occurrence of another energy arrival event while a node is still active is negligible. For simplification, we assume that all energy related events, such as harvesting and consumption, occur at the end of the time slot. Since all nodes perform LPL, any node can capture the transmission of a nearby node as long as it is active. This is described as overhearing. The probability of overhearing a packet is denoted by a Bernoulli process with probability $P_{o}$. With these considerations, we

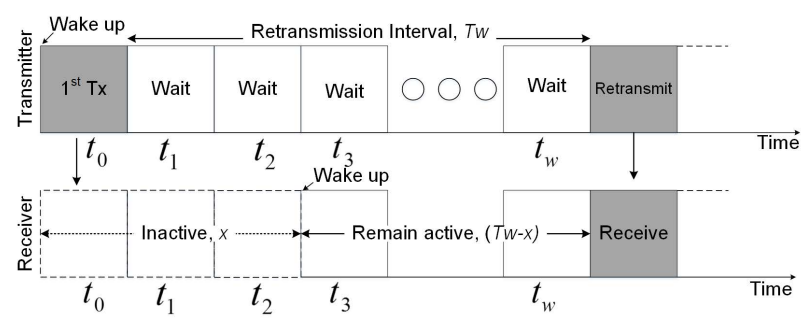

Figure 4: Finding the optimal retransmission interval. formulate the optimal waiting time before retransmission in the following section.

\subsection{Optimal Retransmission Interval}

Once a transmission is unsuccessful (due to the fact that the destination is inactive), a successful rendezvous between a transmitter and receiver requires that the transmitter remains active while the destination wakes up from the sleep state and remains reception capable for at least up to the point where transmitter decided to retransmit. In the following, we develop each of these probabilities for the back-toback transmissions and that using a retransmission interval of $T_{w}$ after a failed transmission attempt.

Probability that transmitter remains active: To find the success probability of a retransmission at a random time, we first find the probability that the source remains active up to that time. Let's consider Fig. 4, where a transmitter node wakes up from inactive state at $t_{0}$ with $E_{h}$ amount of energy. After waking up, it immediately performs a transmission, which is unsuccessful. The transmitter node is now left with $E_{c}=E_{h}-E_{t}$ amount of energy. If the node decides to wait $T_{w}$ time before the next retransmission, it has to have at least $E_{c}-E_{t}$ energy by the end of $T_{w}$ waiting period to perform another transmission. Now, if the transmitter experiences $\eta$ overhearing within $T_{w}$, the following inequality must hold in order for it to be able to retransmit at $\left(t_{w}+1\right)$

$$
\begin{aligned}
& \eta E_{o}+\left(T_{w}-\eta\right) E_{a}^{t} \leq\left(E_{c}-E_{t}\right) \\
& \text { or, } \eta \leq \frac{\left(E_{c}-E_{t}\right)-T_{w} E_{a}^{t}}{\left(E_{o}-E_{a}^{t}\right)}
\end{aligned}
$$

The maximum number of overhearing it will be able withstand to remain retransmission capable is

$$
\eta_{m}^{t}=\left\lfloor\frac{\left(E_{c}-E_{t}\right)-T_{w} E_{a}^{t}}{\left(E_{o}-E_{a}^{t}\right)}\right\rfloor
$$

Now, the probability of remaining capable of transmission after $T_{w}$ is given by the probability of experiencing at most $\eta_{m}^{t}$ overhearing within $T_{w}$, which is

$$
P_{w}^{t}\left(T_{w}\right)=\sum_{i=0}^{\eta_{m}^{t}}\left(\begin{array}{c}
T_{w} \\
i
\end{array}\right) P_{o}^{i}\left(1-P_{o}\right)^{T_{w}-i}
$$

Probability that receiver is active: Successful retransmission also requires that the receiver wakes up in the mean time, and remains capable of reception for at least until thereafter. The probability that the receiver wakes up after $x$ (see Fig. 4) is $P_{e}\left(1-P_{e}\right)^{(x-1)}$. After waking up, the probability that it remains reception capable from $x$ to $t_{w}+1$ 


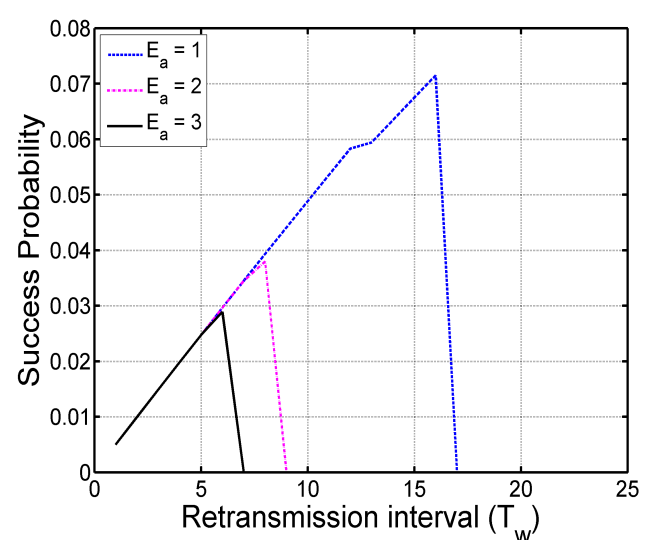

(a)

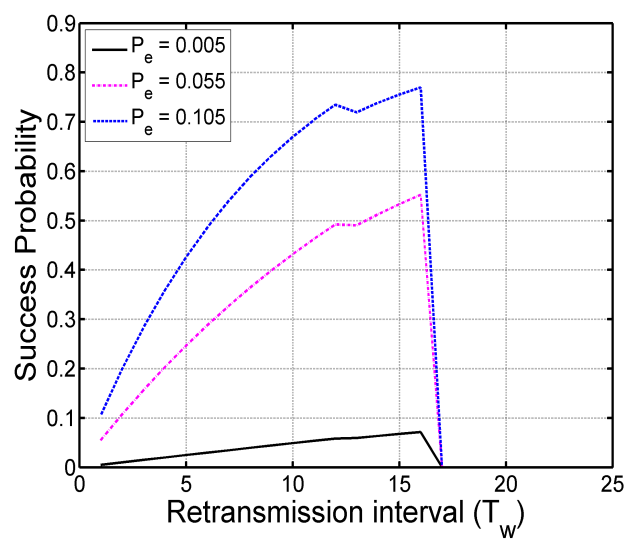

(c)

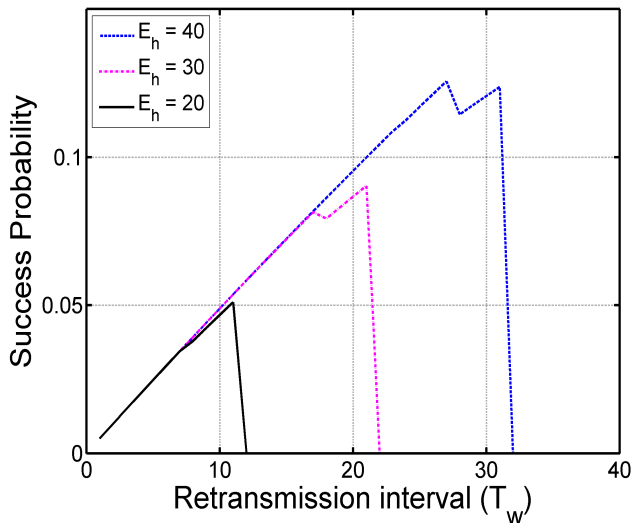

(b)

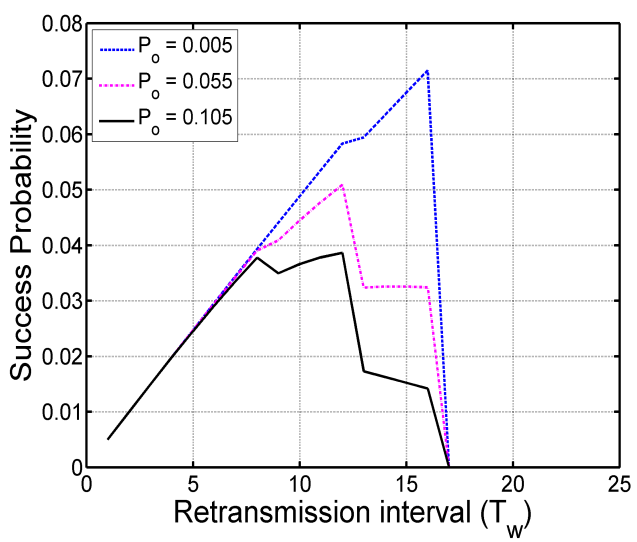

(d)

Figure 5: Probability of success of packet retransmissions in an intermittently connected wireless link using different retransmission intervals.

and beyond can be expressed as

$$
P_{w}^{r}\left(T_{w}+1-x\right)=\sum_{i=0}^{\eta_{m}^{r}}\left(\begin{array}{c}
T_{w}+1-x \\
i
\end{array}\right) P_{o}^{i}\left(1-P_{o}\right)^{T_{w}+1-x-i}
$$

Where

$$
\eta_{m}^{r}=\left\lfloor\frac{\left(E_{h}-E_{r}\right)-\left(T_{w}+1-x\right) E_{a}}{\left(E_{o}-E_{a}\right)}\right\rfloor
$$

\subsubsection{Probability of success with predictive retrans- mission interval}

The probability of a successful retransmission after an interval $T_{w}$ is given by the product of the probabilities of the source remaining capable of transmission up to that time and that the receiver wakes up prior to $T_{w}$ and remains capable of reception afterwards. In summary, the probability of a successful retransmission is

$$
P_{w}^{s}\left(T_{w}\right)=P_{w}^{t}\left(T_{w}\right) \sum_{x=1}^{T_{w}+1}\left[P_{e}\left(1-P_{e}\right)^{(x-1)} P_{w}^{r}\left(T_{w}+1-x\right)\right] P_{c}
$$

Therefore, the maximum success probability achievable with the next retransmission is

$$
P_{m}^{p}=\max _{T_{w}} P_{w}^{s}\left(T_{w}\right) \quad \text { s.t. } 0 \leq T_{w} \leq\left\lfloor\frac{\left(E_{c}-E_{t}\right)}{E_{a}^{t}}\right\rfloor
$$

Consequently, the optimum retransmission interval that maximizes the probability of a successful retransmission is given by

$$
T_{o}^{p}=\arg \max _{T_{w}} P_{w}^{s}\left(T_{w}\right)
$$

To determine the effect of different node parameters on the optimum retransmission interval, we plot the variations of the probability of successful retransmission with a retransmission interval $T_{w}$ for different sets of parameters in Fig. 5. The default parameters for these figures are provided in Table 2. We assume that the energy consumed for transmission, reception, and overhearing are the same, since low power radios require similar amounts of energy to transmit and receive [8]. Therefore, unless otherwise stated, we designate these three events as radio events that consume $E_{r}$ amount of energy. Also, since the energy arrival probability is quite low, we approximate the Binomial distributions of (2) and (3) with Poisson. We also consider $P_{c}=1$ to focus mainly on parameters related to the sensor nodes. 


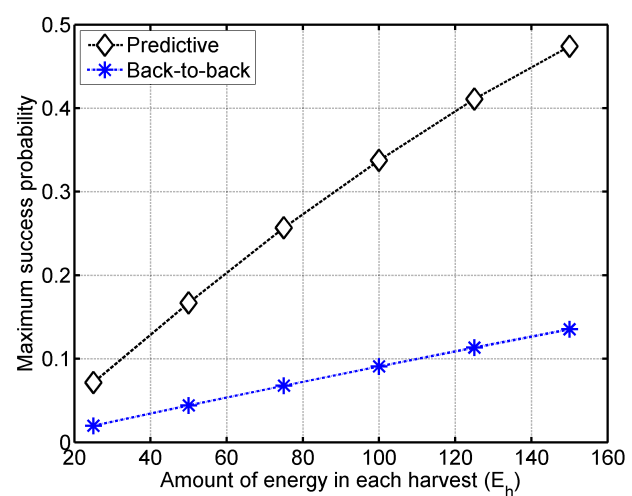

(a)

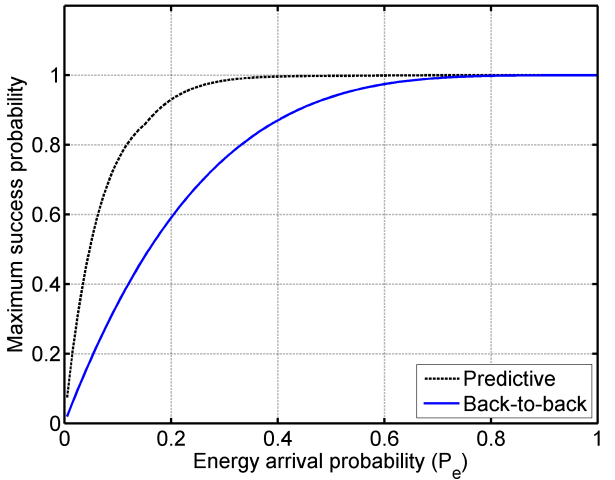

(b)

Figure 6: Maximum success probability achievable using predictive and back-to-back retransmissions in an intermittently connected wireless link.

In Fig. 5 (a) we study the effect of the ambient energy consumption on the optimal retransmission interval. It is observed that the probability of success for retransmissions generally increases with increasing values of $T_{w}$. This is due to the fact that a longer interval allows more likelihood of receiver becoming active in the meantime. However, if the retransmission interval becomes too long, the success probability sharply falls since the transmitter loses energy from ambient activities. It must be noted that when the ambient energy consumption is high, the probability of success is lower. Fig. 5 (b) suggests a smaller waiting period between successive retransmissions if harvested energy is low.

A general observation from these two figures is that it is better to wait until the last moment such that only enough energy for a single transmission is left. For instance, if a node currently has 20 units of energy, each radio events consumes 5 units of energy, and ambient activities constantly consumes 1 unit of energy, a node may wait for next 15 time units before the next retransmission. This maximizes the chances for the receiver to become active while the source still has energy to transmit. However, this observation does not apply for all the cases. For example, Fig. 5 (b) depicts that for $E_{h}=40$, the optimal waiting time becomes 27 instead of 30 . In Fig. 5 (c), we see the variations of the optimal retransmission interval with respect to different energy arrival probabilities. Here, small increase in energy arrival probability has little effect on the optimal delay time, however, the success probability is vastly improved. This is due to the fact that a higher waiting time ensures a higher likelihood of the receiver becoming active regardless of the energy arrival probability. Fig. 5 (d) indicates that the success probability significantly decreases with higher $P_{o}$, which indicates the detrimental effect of higher overhearing.

\subsubsection{Probability of success with back-to-back retrans- missions}

To perform a comparison, we now find the maximum success probability associated with back-to-back retransmissions. If we denote the maximum number of transmission attempts possible by $\beta+1$ after waking up from inactive state with $E_{h}$ energy, and the first transmission was not successful, then we can write

$$
\beta=\left\lfloor\frac{\left(E_{h}-E_{t}\right)}{E_{t}}\right\rfloor
$$

Here, the success probability for each retransmission attempt depends on whether the receiver underwent an energy arrival event in the previous slot and also on the channel condition. The cumulative success probability for retransmission can therefore be written as

$$
P_{m}^{b}=\sum_{i=1}^{\beta} P_{e} P_{c}\left(1-P_{e} P_{c}\right)^{i-1}
$$

We plot the maximum attainable success probability for both back-to-back retransmission and that using predictive retransmission in Fig. 6. It is clear that the predictive retransmission strategy provides a greater probability of success compared to back-to-back transmission policy by leveraging the statistical information at the time of the first transmission and adjusting the retransmission interval accordingly. Moreover, the back-to-back retransmission is a special case for the predictive retransmission scheme when $T_{w}=0$ provides the highest probability of success.

\subsection{Delay Analysis}

We now evaluate the average transmission delay over an intermittently connected wireless link using a specific retransmission scheme. Our approach is to first determine the probability that a packet transmission will be successful in a single active-inactive cycle of the source node. As illustrated in Fig. 7, the average transmission delay is equal to the period of time covering the expected number of active-inactive cycles required before the packet transmission is successful plus the expected delay involved within the active period where the packet is delivered.

The overall probability of success for transmitting a packet in a single active-inactive cycle depends on the probability of success at the very first transmission after waking up from sleep state, and if that is not successful, the maximum success probability achievable from retransmissions. In the following, we first find the success probability of the first transmission, the maximum success probability in a single 
Expected time needed at a successful cycle

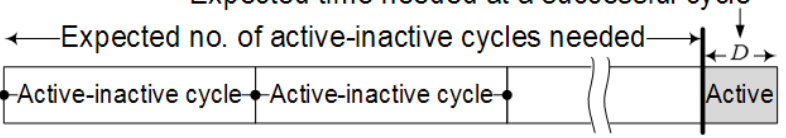

Figure 7: Calculation of the packet transmission delay.

active-inactive period that is achievable using each retransmission policy, and the expected delay involved within an active period to obtain the overall delay.

The success probability of the very first transmission attempt after waking up from a long sleep period depends on whether the receiver is active or not during that transmission. Hence, we find the active probability of the receiver at any random slot.

Let's consider that the receiver has enough energy to be in the active period for an average $\left(\mu_{a}+1\right)$ units of time. Within this time, if an average of $\alpha$ overhearing occurs, we get $\mu_{a} . P_{o}=\alpha$ or, $\mu_{a}=\frac{\alpha}{P_{o}}$. Using a similar approach as (1), we may write

$$
\begin{aligned}
& \alpha E_{o}+\left(\mu_{a}-\alpha\right) E_{a}=E_{h}-E_{r} \\
& \text { or, } \alpha\left(E_{o}-E_{a}\right)+\mu_{a} E_{a}=E_{h}-E_{r} \\
& \text { or, } \mu_{a} \cdot P_{o}\left(E_{o}-E_{a}\right)+\mu_{a} E_{a}=E_{h}-E_{r} \\
& \text { or, } \mu_{a}=\frac{E_{h}-E_{r}}{P_{o}\left(E_{o}-E_{a}\right)+E_{a}}
\end{aligned}
$$

The time between successive energy arrivals is Geometrically distributed with parameter $P_{e}$. Therefore the average interval is $\mu_{e}=\frac{1}{P_{e}}$. When $\mu_{a}$ is much smaller than $\mu_{e}$ (as in the case of intermittently connected network), the probability that the destination is active at a random time slot is approximately $\frac{\left(\mu_{a}+1\right)}{\mu_{e}}$. Hence, the probability of success at the very first transmission can be written as

$$
P_{1} \approx\left[\frac{\left(\mu_{a}+1\right)}{\mu_{e}}\right] P_{c}
$$

Using (4), (5), and (6), we can write the overall success probability at any active-inactive cycle associated with predictive retransmission as

$$
P_{S P}=P_{1} P_{c}+\left(1-P_{1} P_{c}\right) P_{m}^{p}
$$

If a transmission is successful in an active period, the expected time required for the success from the beginning of that active period can be written as

$$
E_{p}[D]=\frac{1}{P_{S P}}\left(P_{1} P_{c}+\left(1-P_{1} P_{c}\right) P_{m}^{p}\left(T_{o}^{p}+1\right)\right)
$$

Similarly, the overall success probability associated with back to back retransmission is

$$
\begin{aligned}
P_{S B} & =P_{1} P_{c}+\left(1-P_{1} P_{c}\right)\left(P_{e} P_{c}\right)+\cdots \\
& +\left(1-P_{1} P_{c}\right)\left(1-P_{e} P_{c}\right)^{\beta-1}\left(P_{e} P_{c}\right) \\
& =P_{1} P_{c}+\left(1-P_{1} P_{c}\right) P_{m}^{b}
\end{aligned}
$$

Consequently, the expected time required for a success
Table 2: Parameters

\begin{tabular}{|l||c|}
\hline Amount of energy in each harvest, $E_{h}$ & 25 \\
\hline Required energy for a radio event, $E_{r}$ & 5 \\
\hline Energy consumption from ambient activities, $E_{a}$ & 1 \\
\hline Probability of an error free channel, $P_{c}$ & 1 \\
\hline Probability of overhearing, $P_{o}$ & 0.005 \\
\hline Energy arrival probability, $P_{e}$ & 0.005 \\
\hline
\end{tabular}

from the beginning of an active period is

$$
\begin{aligned}
E_{b}[D]= & \frac{1}{P_{S B}}\left(P_{1} P_{c}+2\left(P_{e} P_{c}\right)\left(1-P_{1} P_{c}\right)+\cdots\right. \\
+ & \left.\left(1-P_{1} P_{c}\right)\left(1-P_{e} P_{c}\right)^{\beta-1}\left(P_{e} P_{c}\right)(\beta+1)\right) \\
= & \frac{1}{P_{S B}}\left(P_{1} P_{c}+\right. \\
& \left.\sum_{m=2}^{\beta+1} m\left(P_{e} P_{c}\right)\left(1-P_{e} P_{c}\right)^{m-2}\left(1-P_{1} P_{c}\right)\right)
\end{aligned}
$$

The length of an average active-inactive cycle is equivalent to the average energy arrival interval $\mu_{e}$. If we denote the overall success probability at any cycle in general by $P_{s}$ and the expected delay at a successful cycle by $\bar{D}$, we can express the expected overall delay as

$$
\begin{aligned}
E[D]= & \bar{D} P_{s}+\left(\bar{D}+\mu_{e}\right) P_{s}\left(1-P_{s}\right) \\
+ & \left(\bar{D}+2 \mu_{e}\right) P_{s}\left(1-P_{s}\right)^{2}+\cdots \\
= & \bar{D} P_{s}+\bar{D} P_{s}\left(1-P_{s}\right)+\bar{D} P_{s}\left(1-P_{s}\right)^{2}+\cdots \\
& +\mu_{e} P_{s}\left(1-P_{s}\right)+2 \mu_{e} P_{s}\left(1-P_{s}\right)^{2}+\cdots \\
= & \bar{D} \cdot P_{s} \sum_{i=0}^{\infty}\left(1-P_{s}\right)^{i}+\mu_{e}\left(1-P_{s}\right) \sum_{j=1}^{\infty} j P_{s}\left(1-P_{s}\right)^{j-1} \\
= & \bar{D} P_{s} \frac{1}{1-\left(1-P_{s}\right)}+\frac{\mu_{e}\left(1-P_{s}\right)}{P_{s}} \\
= & \bar{D}+\frac{\mu_{e}}{P_{s}}-\mu_{e}
\end{aligned}
$$

To validate our theoretical analysis, we perform simulations written in in $\mathrm{C}++[1]$. It is based on a single sourcedestination pair. Here, both nodes have basic parameters as depicted in Table 2. The source and destination nodes randomly harvest energy and consume that from ambient activities. At time slot 5000, the source generates a packet and inserts that into the transmission queue. We then record the time required to forward the packet to the destination. The simulation is repeated 10000 times and averaged for each observation. We also take into consideration that energy might be harvested while a node is still active. This additional energy is aggregated in an energy buffer. The size of the energy buffer is assumed to be equal to $3 E_{h}$. Results provided in Fig. 8 show that up to $37 \%$ improvement can be achieved through the proposed predictive retransmission scheme.

\section{COOPERATIVE ROUTING WITH PRE- DICTIVE RETRANSMISSION}

In this section, we describe the proposed scheme that applies to predictive retransmission in cooperative routing. When a transmitter's first attempt becomes unsuccessful, it 


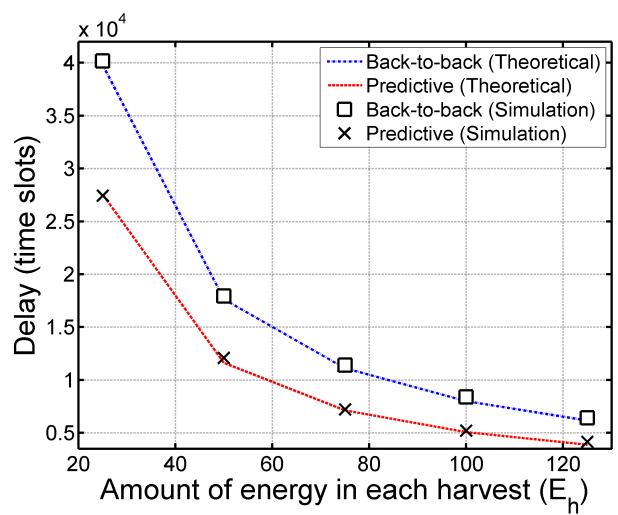

(a)

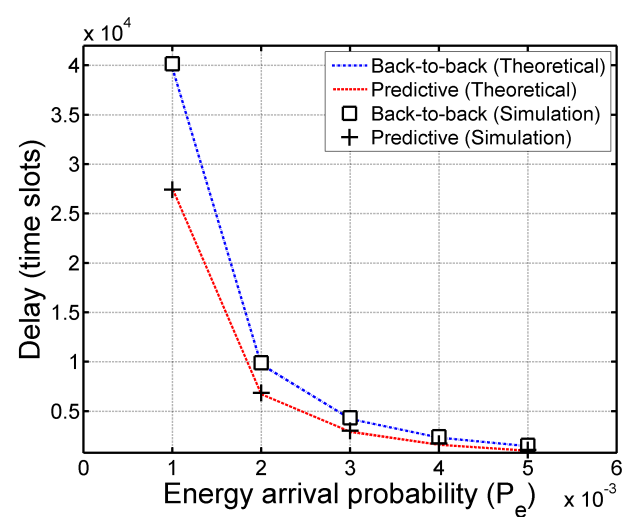

(b)

Figure 8: Delay performance in an intermittently connected wireless link using predictive and back-to-back retransmissions.

calculates it's optimal waiting time $T_{o}^{p}$ before the next retransmission according to it's current energy. If the retransmission is unsuccessful and it is left with some energy, it then finds the next optimum retransmission interval based on its current parameters. This process continues until the transmitter runs out of energy. To facilitate cooperative routing, if any neighboring node overhears a transmission, it captures the packet and determines the intended receiver. If the receiver is also within the transmission range of that node, it waits to see if the receiver acknowledges the transmission from the source. If it does not overhear the acknowledgment, then it assumes that the transmitter's attempt was unsuccessful, and it considers to cooperate in forwarding the packet. The cooperator sets up its own $T_{o}^{p}$ according it's energy buffer to help forward the packet.

To analyze the delay performance of both retransmission schemes with cooperative transmissions, let's consider a scenario where a source and $\kappa$ other cooperators are trying to forward a packet to the receiver. At any given instance, the probability that any node is trying to forward a packet is approximately, $P_{\theta 1} \approx \frac{T_{\theta}}{\mu_{e}}$, where $T_{\theta}=\left(1+T_{o}^{p}+1\right)$, for predictive retransmission, and $T_{\theta}=(1+\beta)$, for back-to-back retransmissions. The probability that at least one among $(\kappa+1)$ nodes attempts to transmit the packet to the receiver can be expressed as

$$
P_{\theta 1}=1-\left(1-P_{\theta 1}\right)^{\kappa+1}
$$

For a single node, the success probability at any random time slot is, $P_{s 1}=\frac{P_{s}}{T_{\theta}}$. Therefore, the overall probability of forwarding the packet with the help of $(\kappa+1)$ nodes at each slot is $P_{s 1} P_{\theta 1}$. Hence, expected delay to successfully deliver the packet is

$$
E\left[\operatorname{Delay}_{(\kappa+1)}\right] \approx \frac{1}{P_{s 1} P_{\theta 1}}
$$

To confirm these results, we use simulations using a similar setup as described in the previous section with the addition of cooperative relays. The results, presented in Fig. 9 , indicate that substantial performance improvement (approximately $30 \%$ ) can be achieved through the predictive retransmission policy over back-to-back retransmissions when cooperative relays are involved.

\section{SIMULATION AND RESULTS}

To evaluate the performance of cooperative transmission with predictive retransmissions in a multi-hop environment, we simulate a sensor network comprising of 25 nodes that are arranged in a uniform grid within a square area of $40 \mathrm{~m} \times 40 \mathrm{~m}$ as depicted in Fig. 10). A sink node is located at coordinate $(40,40)$. A grid spacing of $10 \mathrm{~m}$ is assumed. Nodes are assumed to know their locations. A least-cost routing scheme is applied to determine the minimum hop route from the source to the sink at the network setup phase. The transmission radius was set to $15 \mathrm{~m}$. Therefore, each node has approximately 2 neighbor nodes that have the potential to become cooperators. For instance, node 13 can be assisted by nodes 18 and 14 using cooperative relaying.

At a random time, the node at coordinate $(0,0)$ generates a data packet and attempts to deliver that packet to the sink through multihop routing. We evaluate the end-to-end delays in forwarding the packet for both the back-to-back and predictive retransmission policies, using cooperative transmission in both cases. In order to avoid other delays such as buffer delay, we consider only a single packet flow, i.e. one source. In addition to the parameters provided in Table 2 , we consider here $E_{h}=50$ and $P_{e}=0.001$ to allow diverse simulation setups. Results from the simulations are depicted in Fig. 11. Similar to our findings for a single hop case, it is observed that the end to end delay exponentially decreases with increasing values of the harvested energy (see Fig. 9 (a) for single hop and Fig. 11 (a) for multihop). In Fig. 11 (b), we observe an interesting fact that at high values of $E_{r}$ (e.g., 25), both the back-to-back and predictive retransmission policy provides the same transmission delay. This is expected, since with $E_{r}=25$ and $E_{h}=50$, there is no room left for the predictive policy to wait and choose an optimal retransmission time (here, $E c=50-25=25$ which is barely sufficient for a retransmission but no waiting time). Fig. 11 (c) suggests a linear relationship between the ambient energy consumption and end-to-end delay. All results indicate that the proposed predictive retransmission scheme significantly improves the delay performance of cooperative routing in the ICSN. 


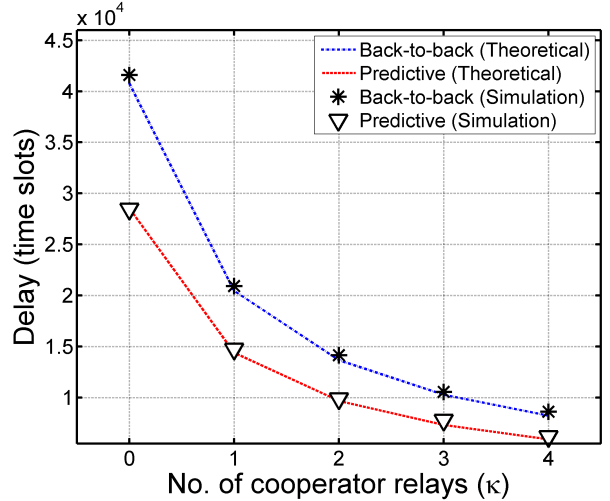

(a)

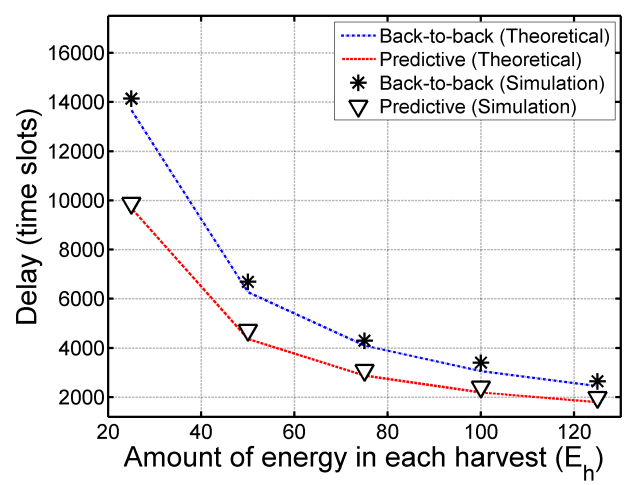

(b)

Figure 9: Delay performance in intermittently connected wireless link using cooperative transmissions with different retransmission policies.

\section{CONCLUSION}

We address the issue of minimizing the end-to-end delay in intermittently connected sensor networks where nodes suffer from random outages due to insufficient energy resources. We propose a scheme by which nodes try to predict retransmission times after each unsuccessful packet transmission that can maximize the probability of successful packet delivery. The predicted retransmission interval is based on the node's current energy level and the characteristics of energy availability. We apply the proposed predictive retransmission scheme to cooperative transmissions to further reduce the transmission delay over intermittently connected wireless links. Analytical and simulation based studies are presented to demonstrate that the proposed scheme significantly reduces the packet transmission delay in intermittently connected networks in comparison to one that uses back-to-back retransmissions.

\section{ACKNOWLEDGMENTS}

This work was supported by NSF grant CNS-1117790.

\section{REFERENCES}

[1] http://webpages.uncc.edu/ ${ }^{\sim}$ anasipur/ICSN/ NetworkCode_Qt.zip. Accessed: 10/23/2015.

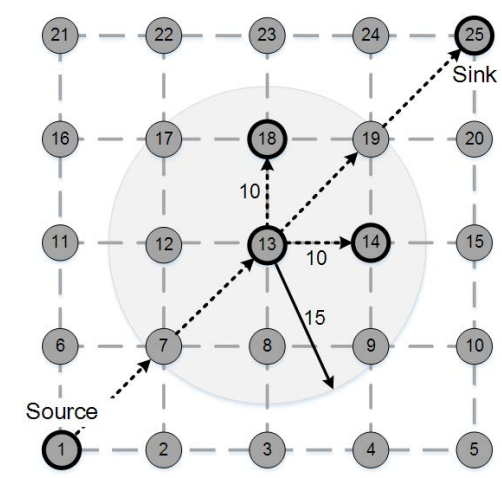

Figure 10: Network model used for simulation.

[2] A. Arora, P. Dutta, S. Bapat, V. Kulathumani, H. Zhang, V. Naik, V. Mittal, H. Cao, M. Demirbas, M. Gouda, et al. A line in the sand: a wireless sensor network for target detection, classification, and tracking. Computer Networks, 46(5):605-634, 2004.

[3] M. Bahrepour, N. Meratnia, M. Poel, Z. Taghikhaki, and P. J. Havinga. Distributed event detection in wireless sensor networks for disaster management. In 2nd International Conference on Intelligent Networking and Collaborative Systems (INCOS), 2010, pages 507-512. IEEE, 2010.

[4] M. Buettner, G. V. Yee, E. Anderson, and R. Han. X-mac: a short preamble mac protocol for duty-cycled wireless sensor networks. In 4th international conference on Embedded networked sensor systems, pages 307-320. ACM, 2006.

[5] H. Chen, L. Cui, and V. O. Li. A joint design of opportunistic forwarding and energy-efficient mac protocol in wireless sensor networks. In Global Telecommunications Conference, pages 1-6. IEEE, 2009.

[6] A. Dunkels. The contikimac radio duty cycling protocol. Technical report, Swedish Institute of Computer Science, 2011.

[7] Z. A. Eu, H.-P. Tan, and W. K. Seah. Opportunistic routing in wireless sensor networks powered by ambient energy harvesting. Computer Networks, 54(17):2943-2966, 2010.

[8] M. Krämer and A. Geraldy. Energy measurements for micaz node. University of Kaiserslautern, Kaiserslautern, Germany, Technical Report KrGe06, 2006.

[9] O. Landsiedel, E. Ghadimi, S. Duquennoy, and M. Johansson. Low power, low delay: opportunistic routing meets duty cycling. In 11th International Conference on Information Processing in Sensor Networks (IPSN), pages 185-196. IEEE, 2012.

[10] Y. Li and R. Bartos. A survey of protocols for intermittently connected delay-tolerant wireless sensor networks. Journal of Network and Computer Applications, 41:411-423, 2014.

[11] S. Liu, K.-W. Fan, and P. Sinha. Cmac: An energy-efficient mac layer protocol using convergent packet forwarding for wireless sensor networks. $A C M$ Transactions on Sensor Networks (TOSN), 5(4):29, 


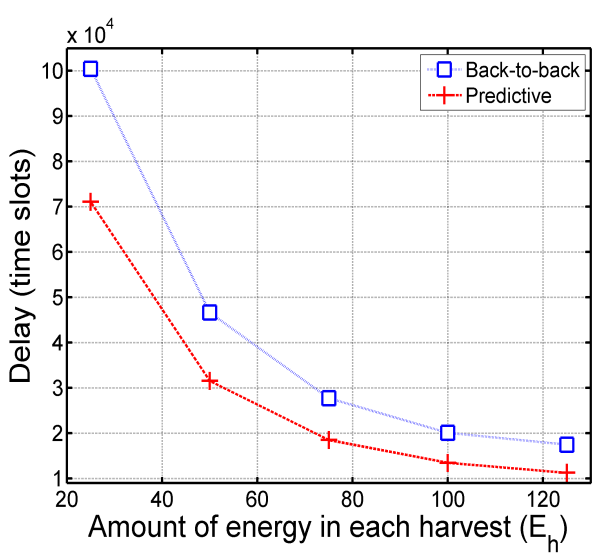

(a)

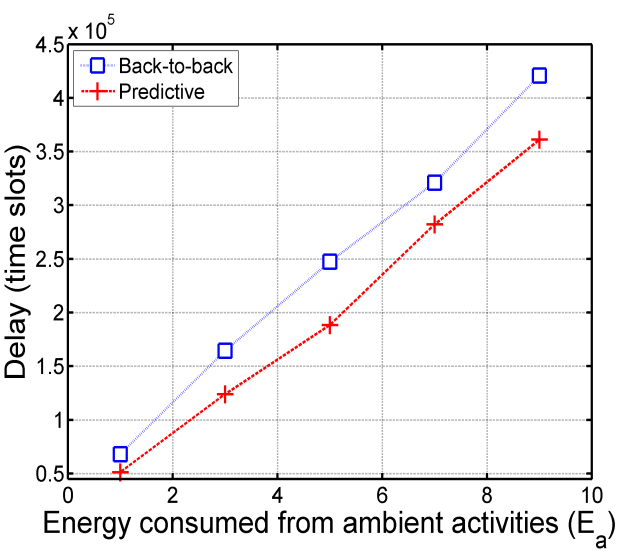

(c)

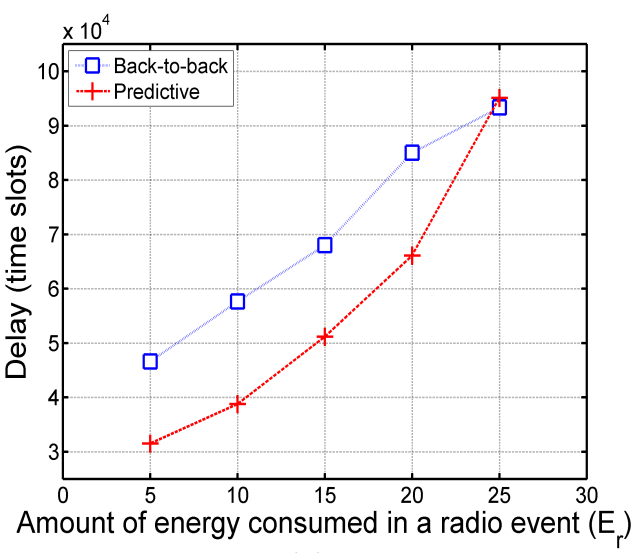

(b)

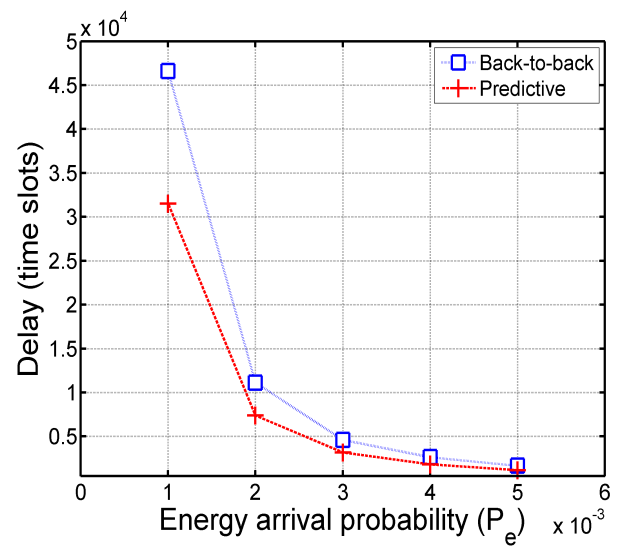

(d)

Figure 11: Performance analysis of cooperative routing under multihop scenario with different retransmission schemes.

2009.

[12] D. Moss and P. Levis. Box-macs: Exploiting physical and link layer boundaries in low-power networking. Computer Systems Laboratory Stanford University, pages 116-119, 2008.

[13] M. Peigney and D. Siegert. Piezoelectric energy harvesting from traffic-induced bridge vibrations. Smart Materials and Structures, (22(9):095019), 2013.

[14] M. M. I. Rajib and A. Nasipuri. Delay performance of intermittently connected wireless sensor networks with cooperative relays. In International Conference on Communication Workshop (ICCW), pages 1994-1999. IEEE, 2015.

[15] S. Sudevalayam and P. Kulkarni. Energy harvesting sensor nodes: Survey and implications. IEEE Communications Surveys and Tutorials, 13(3):443-461, 2011.

[16] L. Tang, Y. Sun, O. Gurewitz, and D. B. Johnson. Pw-mac: An energy-efficient predictive-wakeup mac protocol for wireless sensor networks. In International Conference on Computer Communications (INFOCOM), pages 1305-1313. IEEE, 2011.

[17] G. Werner-Allen, K. Lorincz, M. Ruiz, O. Marcillo,
J. Johnson, J. Lees, and M. Welsh. Deploying a wireless sensor network on an active volcano. IEEE Internet Computing, 10(2):18-25, 2006.

[18] M. Zorzi and R. R. Rao. Geographic random forwarding (geraf) for ad hoc and sensor networks: multihop performance. IEEE Transactions on Mobile Computing, 2(4):337-348, 2003. 